\title{
Diagnostic Tools for the Detection of Subclinical Hepatic Encephalopathy: Comparison of Standard and Computerized Psychometric Tests with Spectral-EEG
}

\author{
Piero Amodio ${ }^{1,3}$, Juan C. Quero ${ }^{2}$, Franco Del Piccolo ${ }^{1}$, Angelo Gatta1, \\ and Solko W. Schalm ${ }^{2}$
}

Received: 10 December 1995; Accepted: 10 June 1996

\begin{abstract}
The prevalence of subclinical hepatic encephalopathy (SHE) varies according to the diagnostic tool used in its detection. Since a standardised approach to the diagnosis of SHE is not yet available, we compared psychometric tests and EEG spectral analysis. On the same day 32 cirrhotic patients without overt hepatic encephalopathy and 18 controls were assessed by psychometric tests, both standard and computerized (CPT), and by EEG spectral analysis (EEG-SA). The CPT, measuring reaction time (Rt) and errors (er), were Font, Choicel, Choice2 and Scan test. The standard psychometric tests were the number connection test (NCT), the Reitan-B test, the Line Tracing Test [for time: LTT(t) and for errors: LTT(er)], and the Symbol Digit test (SD). Both psychometric tests [Reitan-B test, LTT(er) and CPT but Font (Rt) and Choice2 (er)] and EEG-SA parameters [mean dominant frequency (MDF) and theta power $(\theta \%)$ ] significantly correlated $(p<0.05)$ with albumin plasma levels. LTT(er), Scan, Font, Choice1 and Choice2 were significantly related to $\theta \%$ and MDF. There was no control with positive EEG-SA, though one control was positive with $\operatorname{LTT}(t)$ and with the number of errors made during Font and Scan tests. The percentage of cirrhotics with positive EEG-SA was $34 \%\left(\mathrm{Cl}_{95 \%}=19-53\right)$, while $9-66 \%$ were positive with psychometric tests, depending on the test considered. In spite of the correlation between neuropsychological and neurophysiological parameters, the diagnostic agreement between EEG-SA and each psychometric test was not high. In conclusion: 1) neurophysiological and neuropsychological impairment in cirrhotics without overt hepatic encephalopathy were found linked to each other and to hepatic dysfunction; 2) psychometric tests were not sufficiently good predictors of EEG alterations; therefore, neuropsychological tools can not substitute neurophysiological ones to detect CNS dysfunction in liver disease.
\end{abstract}

Keywords: EEG; EEG spectral analysis; psychometric tests; hepatic encephalopathy; liver cirrhosis

1 SplenoEpatologia University of Padova (Italy)

2 Internal Medicine II University Hospital Dijkzigt Rotterdam (The Netherlands)

${ }^{3}$ To whom correspondence should be addressed at Splenoepatologia (Dipartimento di Medicina Clinica e Sperimentale), via Giustiniani, 2, 35128 Padova, Italy 


\section{INTRODUCTION}

Hepatic encephalopathy ( $\mathrm{HE}$ ) is a serious and common complication of liver cirrhosis. It can have overt clinical expression, or be so subtle to be overlooked by routine clinical examination. This condition, which is called subclinical hepatic encephalopathy (SHE), can be detected by psychometric tests, evoked potentials and EEG (Gitlin et al., 1986; Kullmann et al., 1995; Mehndiratta et al., 1990; Parson-Smith et al., 1957; Van der Rijt and Schalm, 1992; Zeneroli et al., 1984).

Zeegen et al. (1970) showed that a psychometric test, the Reitan Trail Making test, was able to detect mental impairment in cirrhotic patients without clinical or EEG findings of encephalopathy. Since then, other psychometric tests have been proposed to detect and measure SHE. Rikkers et al. (1978) suggested that the Wechsler Adult Intelligence Scale (WAIS) performance test, the Reitan-A and B tests, and choice reaction time to light and sound have the best diagnostic values. The usefulness of the WAIS performance group test was later confirmed by Gilberstadt et al. (1980), who found that the most useful tests are the Digit Symbol and the Block Design tests. Also Tarter et al. (1984) showed the efficacy of the Digit Symbol and the Block Design tests, together with the Perdue Pegboard test, for the diagnosis of SHE. Schomerus et al. (1981) emphasised the efficacy of simple and choice reaction times to detect subclinical central nervous system dysfunction in liver cirrhosis.

Another approach to the diagnosis and quantification of SHE is based on the detection of neurophysiological abnormalities. In this regard, EEG, evoked potentials and event evoked responses were used (Davies et al., 1990, Kullmann et al., 1995, Martines et al., 1984, Parson-Smith et al., 1957, Rehnström et al., 1977, Yang et al., 1985). The studies of Rehnström et al. (1977) and Rikkers et al. (1978) showed that psychometric tests are more sensitive than the visual reading of EEG. EEG was also found less sensitive than flash visual evoked responses by Zeneroli et al. (1984). In a comparative study, Mehndiratta et al. (1990) found that the sensitivity of reverse pattern visual evoked responses is lower than that of auditory evoked potentials.

A remarkable finding is that, as yet, there is not a standardised, universally accepted diagnostic tool for SHE.

Nowadays it is easy to set up computerized psychometric tests (CPT) which can measure reaction times and accuracy to perform simple mental tasks. These tests may be simple and repeatable diagnostic tools. However, an accurate comparison between tools used to detect SHE is required.

We therefore performed a study to evaluate the concordance between psychometric tests, either those currently used in the detection of SHE or computerized psychometric tests, with a quantitative neurophysiological diagnostic tool (EEG spectral analysis) in the detection of central nervous system dysfunction in cirrhotics without overt hepatic encephalopathy. 


\section{PATIENTS and METHODS}

We studied 50 subjects: 32 cirrhotics and 18 controls. The diagnosis of cirrhosis was based on case-history, ultrasonography, clinical and biochemical findings. Thirteen cirrhotics and 6 controls were studied in Rotterdam (The Netherlands), the others in Padova (Italy). Cirrhotics age ranged between 25-70 years, with an average age of 56 \pm 10 years (mean \pm sd). Fifteen of them had 5 years of education (level 0 ): 2 in Rotterdam and 13 in Padova; the other 17 had at least 6 years (level 1). All patients stopped alcohol drinking for at least 4 weeks before the study.

According to the Pugh-Child classification (Albers et al., 1989, Merkel et al., 1991), 4 were class A ( 2 from Padova), 27 class B (16 from Padova) and 1 class C (from Padova). Eighteen had alcoholic and 14 non-alcoholic cirrhosis. The prevalence of alcoholic cirrhosis was $62 \%\left(\mathrm{Cl}_{95 \%}=32-86\right)$ in Rotterdam and 53\% $\left(\mathrm{CI}_{95 \%}=29-76\right)$ in Padova. The severity of liver disease, evaluated by Pugh-Child classification, was comparable in alcoholic liver cirrhosis and in non-alcoholic cirrhosis $\left(X^{2}=2.5, \mathrm{p}=\mathrm{n} . \mathrm{s}\right.$. $)$. No cirrhotic patient had overt clinical signs of hepatic encephalopathy. Neurological exam showed, if any, negligible alterations. The main biochemical data of the cirrhotic patients were: total bilirubin $52 \pm 40 \mu \mathrm{mol} / \mathrm{l}$ (mean $\pm \mathrm{sd}$ ), arterial ammonia $62 \pm 25 \mu \mathrm{mol} / \mathrm{l}$, albumin $33 \pm 6 \mathrm{~g} / \mathrm{l}$, Prothrombin time $59 \pm 12 \%$. Patient taking psychotropic drugs were excluded.

Controls were enrolled among paramedical personnel, or patients with minor illness not interfering with mental function, asymptomatic when tests were performed. Other exclusion criteria were alcohol abuse and use of psychotropic drugs.

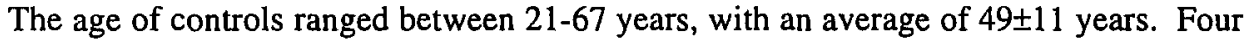
of them had 0 education level, 2 in Rotterdam and 2 in Padova, while the other 14 had 1 education level.

All patients and controls underwent neuropsychological and neurophysiological assessment the same day.

\section{Neuropsycological assessment}

Each patient underwent the NCT (Conn, 1977) (one out of 4 sheet-tests was filled after having filled a different demonstration test), Reitan-B test (Reitan, 1958), LTT (Hamster et al., 1985), SD (Mirsky et al., 1964, Wechsler, 1955), and four CPT: Font, Scan, Choice 1 and Choice 2 test. The CPT were repeated twice. The first session was considered a practice session, thus only the second session was evaluated.

The LTT, belonging to the Beltz Test Gesellschaft (Weinheim), measured the number of errors made and the time spent to draw a line in a bordered trace printed on a sheet without touching the border.

The Scan test (Sternberg, 1969) measured both the number of errors made and the mean reaction time spent to correctly recognize whether there was at least a common digit in pairs of random numbers subsequently displayed for $3 \mathrm{sec}$. on the screen. Subjects were asked to push the digit ' $\mathrm{l}$ ' or ' 3 ' on the keyboard depending on whether they recognized or not common digits among the pairs of numbers subsequently appearing on the screen. 
The Font test (Posner et al., 1969) measured the number of errors and the mean reaction time to correctly recognize if 10 pairs of 2 randomly sorted letters, displayed for $3 \mathrm{sec}$. on the screen, were matched or not. As in the case of the Scan test, answers were given pushing the digit ' 1 ' or ' 3 ' on the keyboard.

The Choice test (Hyman, 1953) was constituted by two blocks of 10 trials. The first block, Choice 1, measured the number of errors and the mean reaction time to correctly recognize a number (comprised between 1 and 4) displayed on the screen for up $3 \mathrm{sec}$ and push the same digit on the keyboard. The second block, Choice2, measured the number of errors and the mean reaction time to correctly recognize a number (comprised between 1 and 4) displayed on the screen for up $3 \mathrm{sec}$ and push the digit in reverse sequence, i.e. pushing $1,2,3$ or 4 when $4,3,2$ or 1 were displayed respectively.

The results of CPT and NCT were considered altered if they were $>2$ standard deviations from the expected values calculated by a predictive model, with age and education as predictors, parameterized in a group of 40 Italian normal subjects. The results of LTT (t) and LTT(er) were considered altered if there score was <-2 (Hamster et al., 1985). The results of SD and Reitan-B test were considered altered if they were below the $<10$ th percentile in the normal population, according to Lezak (1983).

\section{Neurophysiological evaluation}

Spectral EEG analysis was performed according to the method described by Van der Rijt et al. (1984).

In brief, EEG signals from temporo-occipital dipoles (T4-O2 and T3-O1) of patients with closed eyes were recorded, filtering the signal between $0.3-25.6 \mathrm{~Hz}$. The impedance was $<0.5 \mathrm{k} \Omega$, the sampling frequency was $\geq 51.2 \mathrm{~Hz}$, the conversion resolution $5 \mathrm{mV} / 1 \mathrm{l}$ bits. Spectral analysis in the frequency range $1-25.6 \mathrm{~Hz}$, frequency resolution $0.1 \mathrm{~Hz}$, was performed in two periods of $100 \mathrm{sec}$. subdivided in 10 epochs of $10 \mathrm{sec}$. each. The power spectrum was calculated for each $10 \mathrm{sec}$ period using Fast Fourier Transform by means of the original software made in the University of Rotterdam for Olivetti M24 computers (Van der Rijt et al., 1984). The mean power spectrum for each $100 \mathrm{sec}$. period was constructed. The neurophysiological parameters considered were: 1) the mean dominant frequency (MDF) calculated as

$$
\sum_{i=1}^{n}\left(f_{i} S_{i}\right) / \sum_{i=1}^{n} S_{i}
$$

where $f_{\mathrm{i}}=$ frequency $\mathrm{i}$ and $\mathrm{S}_{\mathrm{i}}=$ power of frequency $\mathrm{i}, 2$ ) the relative powers of delta, theta and alpha rhythms, i.e. the percentage power of each rhythm over the power of the total frequency range analysed. Power, a magnitude expressed as $\mathrm{V}^{2}$, reflects the amplitude of EEG waves.

Patients were divided into different stages of $\mathrm{HE}$ depending on their mean dominant frequency (MDF) and the powers of delta and theta $(\theta \%)$ activities, according to Van der Rijt et al. (1984): grade 1 (the lower) EEG alteration was defined as the presence of theta activity $>35 \%$ with normal MDF ( $\geq 6.4 \mathrm{~Hz}$ ), grade 2 alteration was defined as $\mathrm{MDF}<6.4 \mathrm{~Hz}$ with delta $<70 \%$. 


\section{Statistics}

Psychometric test performance times, MDF and $\theta \%$ are expressed as mean $\pm s d$, as they fitted Gaussian distribution. CPT errors were expressed as median and inter-quartile intervals, as they were non-Gaussian distributed values. CPT errors were considered to depend on the probability of a wrong answer in a binomial model with two outcomes: right or wrong answer.

The influence of liver cirrhosis on performance times and CPT errors in psychometric tests was assessed by multivariate regression analysis (Gaussian or logistic, respectively), introducing age, education and cirrhosis as predictors in the model.

The difference in MDF, $\theta \%$ and psychometric test performance time between cirrhotics and controls, and between patients with alcoholic liver cirrhosis and non-alcoholic cirrhosis, was assessed by Student's $t$ test for unpaired data; the number of errors were compared by a non-parametric method (Mann-Whitney test).

Correlations between tests and neurophysiological parameters (MDF and $\theta$ ) were assessed by Gaussian multivariate regression taking into account also age and education as covariates.

The comparison of the frequency of positive psychometric tests in Rotterdam and Padova was carried out by the Fisher's exact test.

Stepwise discriminant analysis was carried out to ascertain the psychometric tests useful to classify cirrhotic patients with or without neurophysiological alterations on EEG spectral analysis.

Statistical analysis was carried out by BMDP statistical package using a computer Compaq Prolinea 4/33.

\section{RESULTS}

\section{Neurophysiological assessment}

A trend for a lower MDF was found in cirrhotics compared to controls (8.3 \pm 1.1 vs. $8.9 \pm 1.2 \mathrm{~Hz}, \mathrm{p}=0.06$ ). $\theta \%$ was significantly and considerably higher in cirrhotics than in controls ( $31.7 \pm 16.5$ vs. $14.7 \pm 6.7 \%, p=0.001)$. Eleven cirrhotics had $\theta \%>35 \%$, and one of them also had MDF $<6.4 \mathrm{~Hz}$. Therefore ten cirrhotics were considered to have class 1 hepatic encephalopathy, and one had class 2 hepatic encephalopathy according to Van der Rijt's EEG classification. The percentage of positive cirrhotics was $34 \%\left(\mathrm{CI}_{95 \%}=19-53\right)$ : $31 \%\left(\mathrm{CI}_{95 \%}=9-62\right)$ in Rotterdam and $37 \%\left(\mathrm{CI}_{95 \%}=16-62\right)$ in Padova. No control had MDF $<6.4 \mathrm{~Hz}$ or $\theta \%>35 \%$.

Patients with alcoholic cirrhosis had MDF and $\theta \%$ comparable to that of patients with non-alcoholic cirrhosis $(8.4 \pm 0.7$ vs. $8.2 \pm 1.3 \mathrm{~Hz}$ and $27 \pm 13$ vs. $35 \pm 1.9 \%$, respectively; $\mathrm{p}=\mathrm{n} . \mathrm{s}$.).

In cirrhotics, MDF and $\theta \%$ significantly correlated with albumin (Figures 1 and 2 ), whereas only $\theta \%$ was weakly correlated with ammonia (Figure 3 ). Age was not found to be a predictor of MDF or $\theta \%$. 


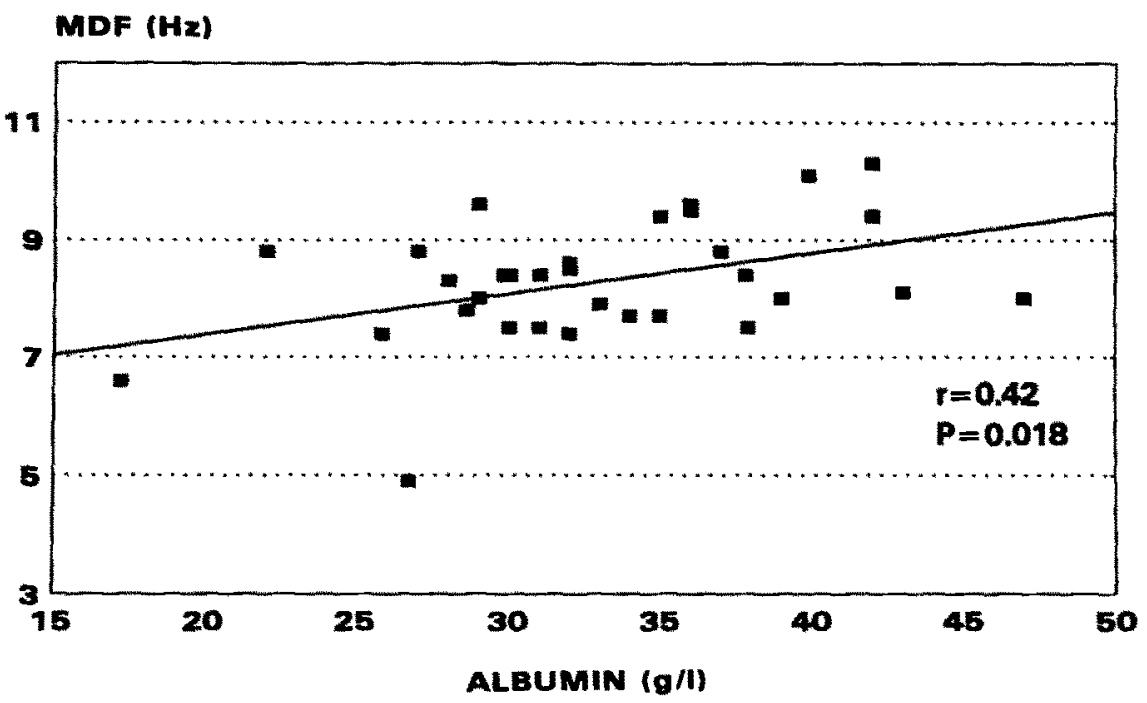

Figure 1. Correlation between MDF and albumin plasma levels.

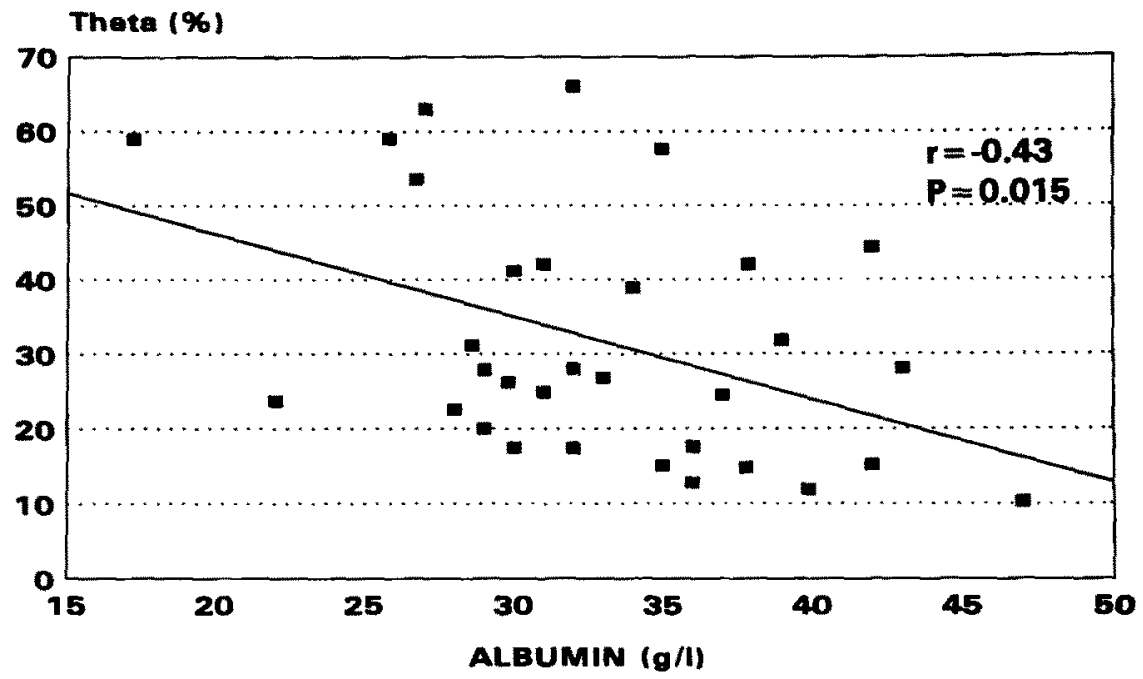

Figure 2. Correlation between theta relative power and albumin plasma levels. 


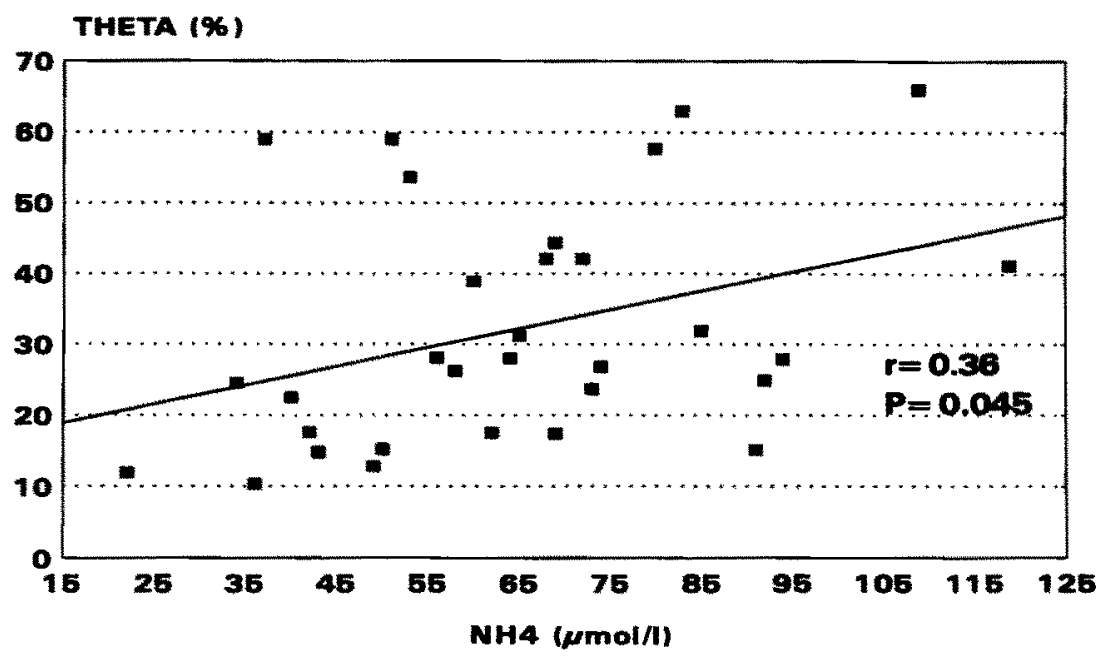

Figure 3. Correlation between theta relative power and plasma ammonia levels.

\section{Neuropsychological assessment}

In mean, all psychometric tests were performed significantly more slowly in cirrhotics than in controls, with the exception of LTT which was only marginally altered (Table 1).

The number of errors made during the Scan and Choice2 tests was higher in cirrhotics, whereas the errors done performing Font and Choicel test were not significantly higher (Table 1). The reaction time of Choicel and Scan test were higher in alcoholic liver cirrhosis than in non-alcoholic cirrhosis $(Z=1.6 \pm 1.7$ vs. $0.07 \pm 1.05 ; \mathrm{p}<0.01$ and $1.25 \pm 1.8$ vs. $0.03 \pm 1.2 ; p<0.05)$. The other psychometric tests did not differ between alcoholic and non-alcoholic liver cirrhosis.

Even considering age and education as covariates which may interfere with psychometric lest results, cirrhosis was found to be an independent predictor influencing all psychometric tests, with the exception of LTT(t), Font (er) and Choicel (er).

The prevalence of positive psychometric tests ranged between $9-66 \%$, depending on the test considered, and it was different in Dutch cirrhotic patients and Italian cirrhotic patients, particularly as regarded Reitan-B test, DS and LTT (Table 2).

All psychometric tests but NCT, LTT (t), SD, Choice2 (Rt), and Font (Rt) were found correlated with the severity of liver failure expressed by albumin plasma levels; no test was found correlated with ammonia plasma levels (Table 3 ). 
Table 1. Comparison of psychometric tests in controls and cirrhotics.

\begin{tabular}{lccc}
\hline Test & Cirrhotics & Controls & P \\
\hline NCT (sec) & $52 \pm 24$ & $29 \pm 8$ & $<0.001$ \\
Reitan-B (sec) & $144 \pm 76$ & $75 \pm 28$ & $<0.001$ \\
LTT (t) (sec) & $122 \pm 44$ & $99 \pm 32$ & 0.06 \\
LTT (er) (n. err.) & $101 \pm 78$ & $26 \pm 13$ & $<0.001$ \\
DS (items) & $27 \pm 16$ & $49 \pm 9$ & $<0.001$ \\
Scan Rt (msec) & $1586 \pm 374$ & $1163 \pm 249$ & $<0.001$ \\
Scan er* & $9.5(13.25)$ & $4(4.5)$ & $<0.001$ \\
Font Rt (msec) & $1197 \pm 653$ & $802 \pm 293$ & 0.002 \\
Font er* & $0(0.75)$ & $0(0.25)$ & 0.39 \\
Choicel Rt (msec) & $1152 \pm 266$ & $818 \pm 132$ & $<0.001$ \\
Choicel er* & $0(1)$ & $0(0)$ & 0.15 \\
Choice2 Rt (msec) & $1442 \pm 424$ & $1053 \pm 203$ & $<0.001$ \\
Choice2 er* & $1(2)$ & $0(1)$ & $<0.001$ \\
\hline
\end{tabular}

The number of errors is expressed as median (inter quartile intervals), and compared by a nonparametric method (Mann-Whitney test) after rank conversion.

Table 2. Percentage of positive psychometric tests in cirrhotic patients in Padova and Rotterdam.

\begin{tabular}{lcccc}
\hline Test & Total & Padova & Rotterdam & P§ \\
\hline NCT & $28(14-47)^{*}$ & $37(16-62)$ & $15(2-45)$ & 0.24 \\
Reitan B & $22(9-40)$ & $37(16-62)$ & $0(0-25)$ & 0.03 \\
LTT (t) & $19(7-36)$ & $32(13-56)$ & $0(0-25)$ & 0.06 \\
LTT (er) & $66(47-81)$ & $84(60-97)$ & $38(14-68)$ & 0.02 \\
SD & $53(35-71)$ & $74(49-91)$ & $23(5-54)$ & 0.01 \\
Font (Rt) & $28(14-47)$ & $21(6-46)$ & $38(14-68)$ & 0.42 \\
Font (er) & $12(4-29)$ & $5(0-26)$ & $23(5-54)$ & 0.27 \\
Scan (Rt) & $19(7-36)$ & $27(9-51)$ & $8(2-36)$ & 0.36 \\
Scan (er) & $37(21-56)$ & $32(13-56)$ & $46(19-75)$ & 0.47 \\
Choice1 (Rt) & $19(7-36)$ & $16(3-40)$ & $23(5-54)$ & 0.66 \\
Choice1 (er) & $16(5-33)$ & $21(6-46)$ & $8(0-36)$ & 0.62 \\
Choice2 (Rt) & $9(2-25)$ & $10(1-33)$ & $8(0-36)$ & 0.99 \\
Choice2 (er) & $19(7-36)$ & $21(6-46)$ & $18(2-46)$ & 0.99 \\
\hline
\end{tabular}

* $\mathrm{Cl}_{95 \%}$. Fisher's exact test, 2-tail. 
Table 3. Correlations of psychometric tests with plasma albumin and ammonia levels in cirrhotic patients.

\begin{tabular}{lcccccc}
\hline & \multicolumn{3}{c}{ Albumin plasma level } & \multicolumn{3}{c}{ Ammonia plasma level } \\
Test & coeff & $r$ & $P$ & coeff & $r$ & $P$ \\
\hline NCT & -1.09 & -0.28 & 0.11 & -0.003 & -0.003 & 0.98 \\
Reitan B & -5.4 & -0.44 & 0.01 & 0.35 & 0.11 & 0.56 \\
LTT (t) & 1.4 & 0.2 & 0.27 & -0.09 & -0.047 & 0.80 \\
LTT (er) & -5.7 & -0.46 & 0.007 & -0.34 & -0.10 & 0.60 \\
SD & 0.80 & 0.31 & 0.08 & -0.14 & -0.14 & 0.43 \\
Font (Rt) & -27 & -0.27 & 0.13 & -1.08 & 0.11 & 0.57 \\
Font (er*) & -0.079 & & $<0.001$ & -0.008 & & 0.40 \\
Scan (Rt) & -31 & -0.52 & 0.0019 & -1.6 & -0.06 & 0.6 \\
Scan (er*) & -0.10 & & $<0.001$ & 0.0002 & & 0.99 \\
Choice1 (Rt) & -24 & -0.56 & $<0.001$ & -0.67 & 0.05 & 0.86 \\
Choice1 (er*) & -0.11 & & $<0.001$ & 0.0035 & & 0.70 \\
Choice2 (Rt) & -2.1 & -0.03 & 0.8 & 2.3 & 0.12 & 0.51 \\
Choice2 (er*) & -0.11 & & $<0.001$ & 0.0002 & & 0.46 \\
\hline
\end{tabular}

\section{Relationship between EEG spectral analysis and psychometric tests}

In cirrhotics MDF was inversely related to all psychometric tests, with the exception of NCT and LTT(t) (Table 4).

$\theta \%$ was found directly correlated with LTT(er), Scan (Rt), Scan (er), Font (er), Choice1 (Rt), Choice1 (er) and Choice2 (er) (Table 4).

However, the diagnostic concordance between EEG-SA and psychometric tools in the detection of SHE in cirrhotics was not complete (Table 5). On the contrary, the diagnostic concordance in controls was higher: no control was positive on EEG-SA and only 3 psychometric tests gave one false positive result each in controls: LTT(t), Font (Rt), Font (er), Scan (er).

The sole Reitan-B test was found useful to discriminate cirrhotic patients with or without neurophysiological alterations on EEG-SA ( $F=6.16 \mathrm{P}=0.018$ ) 
Table 4. Correlations of MDF and $\%$ with psychometric tests in cirrhotics.

\begin{tabular}{lcccc}
\hline & \multicolumn{2}{c}{ MDF } & \multicolumn{2}{c}{$\%$} \\
Test & coeff & $P$ & coeff & $P$ \\
\hline NCT & -4.1 & 0.24 & -0.10 & 0.65 \\
TMT B & -35 & 0.002 & 0.92 & 0.23 \\
LTT (t) & 1.42 & 0.8 & -0.71 & 0.12 \\
LTT (er) & -36 & 0.006 & 2.01 & 0.015 \\
SD & 4.23 & 0.06 & 0.03 & 0.86 \\
Font (Rt) & -408 & 0.0002 & 14 & 0.07 \\
Font (er*) & -0.85 & $<0.001$ & 0.032 & 0.016 \\
Scan (Rt) & -205 & 0.0006 & 9.8 & 0.01 \\
Scan (er*) & -0.30 & $<0.001$ & 0.019 & $<0.001$ \\
Choicel (Rt) & -110 & 0.016 & 6.8 & 0.016 \\
Choicel (er*) & -0.87 & $<0.001$ & 0.048 & $<0.001$ \\
Choice2 (Rt) & -170 & 0.002 & 4.35 & 0.23 \\
Choice2 (er*) & -0.30 & 0.011 & 0.028 & $<0.001$ \\
\hline
\end{tabular}

* Logistic regression

Table 5. Values of psychometric tests as predictors of EEG alterations.

\begin{tabular}{lcccc}
\hline Test & Sensitivity $(\%)$ & Specificity (\%) & PPV (\%) & NPV (\%) \\
\hline NCT & 45 & 81 & 55 & 74 \\
Reitan B & 45 & 90 & 71 & 90 \\
LTT (t) & 36 & 90 & 67 & 73 \\
LTT (er) & 73 & 62 & 38 & 73 \\
SD & 64 & 52 & 41 & 73 \\
Font (Rt) & 45 & 80 & 55 & 74 \\
Font (er) & 18 & 90 & 50 & 68 \\
Scan (Rt) & 36 & 90 & 67 & 90 \\
Scan (er) & 45 & 66 & 42 & 70 \\
Choicel (Rt) & 27 & 86 & 50 & 69 \\
Choicel (er) & 27 & 90 & 60 & 70 \\
Choice2 (Rt) & 9 & 90 & 33 & 65 \\
Choice2 (er) & 18 & 81 & 33 & 65 \\
\hline
\end{tabular}




\section{DISCUSSION}

EEG evaluation by spectral frequency analysis, performed according to Van der Rijt (1984), quantified MDF and $\theta \%$. This last parameter distinguished cirrhotic patients from controls, as groups, better than MDF. Cirrhotic patients with abnormally high $\theta \%(>35 \%)$ and $\mathrm{MDF}>6.4 \mathrm{~Hz}$ were thought to present the first neurophysiological signs of central nervous system dysfunction due to hepatic failure (Van der Rijt et al., 1984). Therefore, they were considered to suffer from subclinical hepatic encephalopathy.

No control had such neurophysiological features; whereas Conn's EEG grading (Conn et al., 1977) which would have classified 12 cirrhotics as anomalous, would also have classified 3 controls as anomalous (score 1).

The significant correlation of $\theta \%$ with albumin and ammonia in our series was in keeping with the opinion that this neurophysiological parameters is a marker of hepatic encephalopathy. In fact, a relationship has already been found between albumin plasma level and mental derangement in cirrhotics (Gilberstadt, 1980, Schomerus et al, 1981). Low albumin plasma levels reflect liver dysfunction and/or may increase the unbound form of substances altering CNS function. In fact, the unbound, free form of such substances might easily enter blood-brain barrier. For example, free tryptophan, the precursor of the inhibitor neurotransmitter serotonin, is high in cirrhosis, even though total plasma levels do not increase (Ono et al., 1978). Similar mechanisms could also apply to benzodiazepine-like substances, possibly involved in hepatic encephalopathy (Jones et al., 1989).

On the average, psychometric performance of cirrhotic patients was significantly lower than that of controls for all tests, with the exception of LTT and the number of errors of Font and Choicel tests. Font and Choicel tests were the least demanding of the CPT; therefore they could be too simple to detect cirrhotics with subtle mental impairment. Also concerning the LTT, the speed to draw a line with a pencil could be less demanding than the accuracy to draw it, at least for cirrhotic patients.

As it was found for EEG-SA parameters, low cognitive performance was linked to the reduction of albumin plasma levels, thus suggesting a relationship with liver disease and/or the free form of CNS inhibitors. The lack of correlation between psychometric tests and ammonia was rather disturbing: a correlation of psychometric tests and ammonia was found by Rikkers et al. (1978). However, it was not confirmed by Gilberstadt (1980). It is plausible that such a correlation needs a higher number of subjects to be found because the relationship between ammonia plasma levels and hepatic encephalopathy is not close. In fact, other toxic substances can modulate ammonia toxicity (Zieve, 1981). The finding that $\theta \%$, but not psychometric tests, were correlated with ammonia plasma levels may suggest that neuropsychological parameters are less effective in detecting central nervous system dysfunction due to liver disease.

The prevalence of psychometric alterations widely varied depending on the test used. Moreover, the prevalence of alterations was clearly different in Dutch and Italian patients, suggesting a cultural effect. In fact, both the prevalence of alcoholic abuse and the severity of liver disease were similar in the two groups. The only clear difference was the education 
level which was lower in Italian cirrhotics, particularly in those belonging to the older age group (they attended school when compulsory education in Italy was five years only).

In our opinion, this could be the reason why psychometric tests standardised only by age (Reitan-B test, LTT, DS) clearly showed a higher prevalence of altered results in Italian than in Dutch cirrhotics, whereas the results of CPT and NCT, which were standardised by age and education level, were less heterogeneous. This finding emphasises the need to be cautious when comparing psychometric data without taking into account confounders such as age, education and, possibly, cultural background.

The significant correlation of neurophysiological parameters with neuropsychological parameters reflecting CNS impairment in cirrhotics without overt hepatic encephalopathy has never been so clearly shown, at least to our knowledge. Possibly, the detection of such a relationship was made easier by the use of quantified EEG analysis, which permitted the exact measuring of EEG parameters.

Even if correlated with neurophysiological parameters, the diagnostic concordance of psychometric tests with EEG-SA in the detection of cirrhotic patients with subclinical hepatic encephalopathy was not high. The sole Reitan-B test seemed efficient to discriminate between cirrhotic patients with or without EEG-SA alterations. However, its results were possibly influenced by cultural factors.

The low concordance between psychometric and neurophysiological parameters may be due to: 1) not yet well-defined cut-off limits for EEG-SA and psychometric tests; 2) incomplete parallelism between neuropsychological and neurophysiological consequences of CNS dysfunction due to liver failure.

These findings suggest that further research is needed to clarify the relationship between neurophysiological and neuropsychological features of subclinical hepatic encephalopathy. Moreover, diagnostic criteria based also on the prognostic value of neurophysiological and neuropsychological alterations should be developed. In the meantime, our data suggest that complete assessment of cirrhotic patients for subclinical hepatic encephalopathy needs both neurophysiological and psychometric approach, as neither of them gives exhaustive information.

\section{ACKNOWLEDGMENTS}

This work was supported in part by the Italian Liver Foundation. The authors thank Mrs. Antonietta Sticca and Mr. Orfeo Griggio for technical support.

\section{REFERENCES}

Albers, I., Hartmann, H., Bircher, J., and Creutzfeldt, W. (1989). Superiority of the Child-Pugh classification to quntitative liver function tests for assessing prognosis of liver cirrhosis. Scand. J. Gastroenterol. 24:269276.

Conn, H.O. (1977). Trailmaking and Number-Connection Tests in the assessment of mental state in portal systemic encephalopathy. Dig. Dis. 22:541-550.

Conn, H.O., Leevy, C.M., Vlahcevic, Z.R., Rodgers, J.B., Maddrey, W.C., Seeff, L., and Levy, L.L. (1977). A comparison of lactulose and neomycin in the treatment of chronic portal-systemic encephalopathy: A double-blind controlled trial. Gastroenterology 72:573-583. 
Davies, M.G.,. Rowan, M.J., and Feely, J. (1990). Flash visual evoked responses in the early encephalopathy of chronic liver disease. Scand. J. Gastrolenterol. 25:1205-1214.

Gilberstadt, S.I., Gilberstadt, H., Zieve, L., Bougel, B., Collir, R.Y., and Mc Clain, C.J. (1980). Psychomotor performance defects in cirrhotics patients without overt encephalopathy. Arch. Intern Med. 140:519-521.

Gitlin, N., Lewis, D.C., and Hinkley, L. (1986). The diagnosis and prevalence of subclinical hepatic encephalopathy in apparently healthy, ambulant, non-shunted patients with cirrhosis. J. Hepatol. 3:75-82.

Hamster, W., Kluck, M.; and Schomerus, H. (1985). PSE-Syndrome tes. Kurtzform. Duphar Pharma GmbH, Hannover.

Hyman, R. (1953). Stimulus information as a determinant of reaction time. J. Exper. Psychol. 45:188-196.

Jones, E.A., Skolnick, P., Gammal, S.H., Basile, A.J., and Mullen, K.D (1989). The $\gamma$-amino butyrric acid A $\left(\mathrm{GABA}_{\mathrm{A}}\right)$. Receptor Complex and Hepatic Encephalopathy. Some recent advances. Ann. Int. Med. 110:532-546.

Kullmann, F., Hollerbach, S., Holstege, A., and Schölmerich. J. (1995). Subclinical hepatic encephalopathy: the diagnostic value of evoked potentials. I. Hepatol. 22:101-110.

Lezak, M.D. (1983). A compendium of tests and assessment techniques. In (M.D. Lezak, ed). Neuropsychological Assessment. Oxford University Press, 1983.

Martines, D., Martini, A., Battaglia, G., Gerunda, G., Comacchio, F., Vespasiani, F., and Gastaldon, A. (1984). Brain-stem auditory-evoked responses (BAERS) in the clinical evaluation of hepatic encephalopathy. In (G. Kleinberg, P. Ferenci, P. Rieder, eds). Advances in Hepatic Encephalopathy and Urea Cycle Diseases. Karger, Basel , pp. 430-435.

Mehndiratta, M.M., Sood, GK, Sarin, S.K., and Gupta, M. (1990). Comparative evaluation of visual, somatosensory, and auditory evoked potentials in the detection of subclinical hepatic encephalopathy in patients with non alcoholic cirrhosis. Am. J. Gastroenterol. 85:799-803.

Merkel, C., Gatta, A.., Zoli, M., Bolognesi, M., Angeli, P., Iervese, T., Marchesini, G., and Ruol, A. (1991). Prognostic value of galactose elimination capacity, aminopyrine breath test, and ICG clearance in patients with cirrhosis. Comparison with the Pugh score. Dig. Dis. Sci. 36:1197-1203.

Mirsky, A., and Kornetsky, C. (1964). On the dissimilar effects of drugs on the digit symbol substitution and continuous performance test. Psychopharmacologia 5:161-177.

Ono, J., Hutson, D.G., Dombro, R.S., Levi, J.U., Livingstone, A., and Zappa, R. (1978). Tryptophan and hepatic coma. Gastroenterology 74:196-200.

Parson-Smith, B.G., Summerskill, W.HJ., Dawson, A.M., and Sherlock, S. (1957). The electroencephalograph in liver disease. Lancet 2:867-871.22.

Posner, M.I., Boies, S.J., Eichelman, W.H., and Taylor, R.L. (1969), Retention of visual and name codes of single letters. J. Exper. Psychol. Monogr. 79 (1 part 2):1-16.

Rehnström, S., Simert, G., Hasson, J.A., Johanson, G., and Vang, J. (1977). Chronic hepatic encephalopathy - A psychometrical study. Scand. J. Gastroenterol. 12:305-311.1S.

Reitan, R.M. (1958). Validity of the trail making test an indicator of organic brain damage. Percept. Mot. Skills 8:271-276.

Rikkers, J., Jenku, P., Rudman, D., and Freides, D. (1978). Subclinical hepatic encephalopathy: detection, prevalence, and relationship to nitrogen metabolism. Gastruenterology 75:462-469.

Schomerus, H., Hamster, W., Blunck, H., Reinhard, U., Mayer, K., and Dolle. W. (1981). Latent portasystemic encephalopathy. Nature of cerebral functional defects and their effect on fitness to drive. Dig. Dis. Sci. 27:622-630.

Sternberg S. (1969). Memory-scanning: mental processes. Am. Scient. 57:4:421-457.

Tarter, R.E., Hegedus, A.M., Van Thiel, D.H., Schade, R.R., Galaver, G.S., and Starzl, T.E. (1984). Nonalcoholic cirrhosis associated with neuropsycological dysfunction in absence of overt evidence of hepatic encephalopathy. Gastroenterology 86:1421-1427.

Van der Rijt, C.C.D., Schalm, S.W., Groot, G.M., and de Vliger, M. (1984). Objective measurement of hepatic encephalopathy by means of automated EEG analysis. Electroenceph. Clin. Neurophysiol. 57:423-426.

Van der Rijt, C.C.D., and Schalm, S.W. (1992). Quantitative EEG analysis and evoked potentials to measure (latent) hepatic encephalopathy. $J$. Hepatol. 14:141-142.

Wechsler, D. (1955). Wechsler Adult Intelligence Scale. New York: Psychological Corporation.

Yang, S.S., Chu, N.S., and Liaw, Y.F. (1985). Somatosensory evoked potentials in hepatic encephalopathy. Gastroenterology 89:625-630.

Zeegen, R., Drinkwater, J.E., and Dawson, A.M. (1970). Method for measuring cerebral dysfunction in patients with liver disease. Br. Med. J. 2;633-636.

Zeneroli, M.L., Rinelli, G., Gollini, G., Penne, A., Messori, E., Zani, G., and Ventura, E. (1984). Visual evoked potentials: a diagnostic tool for the assessment of hepatic encephalopathy. Gut 25:291-299.

Zieve, L. (1981). The mechanism of hepatic coma. Hepatology 1:360-65. 